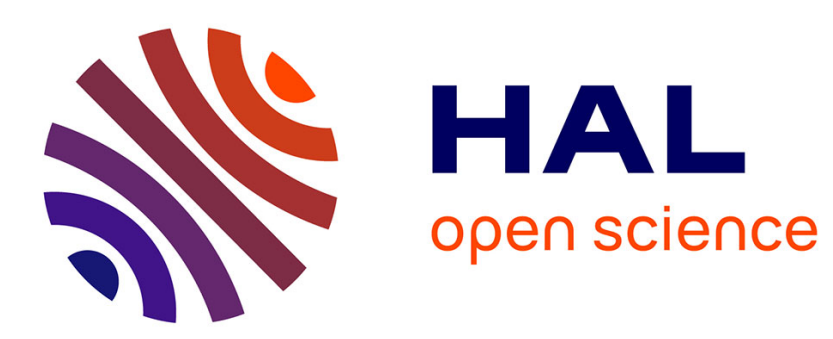

\title{
Intervalle de confiance de la signature obtenue par D.L.T.S., d'un piège profond
}

\author{
G. Sagnes, G. Bastide, H. Lim
}

\section{To cite this version:}

G. Sagnes, G. Bastide, H. Lim. Intervalle de confiance de la signature obtenue par D.L.T.S., d'un piège profond. Revue de Physique Appliquée, 1982, 17 (12), pp.787-792. 10.1051/rphysap:019820017012078700 . jpa-00245058

\section{HAL Id: jpa-00245058 https://hal.science/jpa-00245058}

Submitted on 1 Jan 1982

HAL is a multi-disciplinary open access archive for the deposit and dissemination of scientific research documents, whether they are published or not. The documents may come from teaching and research institutions in France or abroad, or from public or private research centers.
L'archive ouverte pluridisciplinaire HAL, est destinée au dépôt et à la diffusion de documents scientifiques de niveau recherche, publiés ou non, émanant des établissements d'enseignement et de recherche français ou étrangers, des laboratoires publics ou privés. 
Classification

Physics Abstracts

$71.55 \mathrm{Fr}$

\title{
Intervalle de confiance de la signature obtenue par D.L.T.S., d'un piège profond
}

\author{
G. Sagnes, G. Bastide et H. Lim \\ Université des Sciences et Techniques du Languedoc, \\ Centre d'Etudes d'Electronique des Solides, associé au C.N.R.S., \\ Place E. Bataillon, 34060 Montpellier Cedex, France
}

(Reçu le 10 mars 1982, révisé le 7 juillet 1982, accepté le 13 septembre 1982)

\begin{abstract}
Résumé. - Nous donnons à partir des résultats d'une simulation numérique, une méthode statistique de détermination des intervalles de confiance des mesures de l'énergie d'activation et de la section de capture d'un piège profond par D.L.T.S. A partir des résultats de la simulation, nous déterminons deux relations empiriques qui permettent dans leurs limites de validité, de calculer rapidement ces intervalles.
\end{abstract}

\begin{abstract}
In this paper, we study a numerical simulation method with which one can determine the confidence intervals of activation energy and capture cross-section of a deep level measured by D.L.T.S. method. From the results of this simulation, we determine two empirical relations and their limits of validity, which permit to calculate easily these intervals.
\end{abstract}

1. Introduction. - Les propriétés électriques et optoélectroniques potentielles d'un matériau semiconducteur sont fortement liées à la présence de défauts localisés dans le réseau cristallin. Ceux-ci peuvent être introduits accidentellement avec les produits dopants lors de la fabrication du matériau ou être liés à des défauts de structure du réseau. Ils se comportent comme des centres pièges à porteurs et peuvent donc modifier notablement les propriétés électriques et optoélectroniques du matériau.

La technique la plus performante de détection de ces centres a été proposée par D. V. Lang [1] en 1974 sous la dénomination « Deep Level Transient Spectroscopy ", D.L.T.S. Elle consiste à déterminer la constante de temps de vidage d'un niveau profond (Temps d'émission d'un porteur par le centre) dans la zone de charge d'espace vide de porteurs libres d'une jonction p-n ou Schottky polarisée dans le sens bloquant, le remplissage du niveau (capture d'un porteur par le centre) est préalablement assuré par une impulsion électrique déblocante. Alors, on montre [1] que la variation de la capacité transitoire de la jonction pendant l'émission des porteurs par le piège est une exponentielle dont la constante de temps est égale à celle, $\tau_{\mathrm{e}}$, d'émission du piège excité qui est donnée par l'expression :

$$
\frac{1}{\tau_{\mathrm{e}}}=A T^{2} S \exp (-E / k T)
$$

où $T$ est la température, $k$ la constante de Boltzmann et $A=3,25 \times 10^{25} \mathrm{~m}^{*} / \mathrm{m}_{0}\left(\mathrm{~m}^{-2} \cdot \mathrm{s}^{-1} \cdot \mathrm{K}^{2}\right)$ un paramètre qui ne dépend que de la masse effective du matériau.

La détermination de la signature caractéristique du piège, c'est-à-dire son énergie d'activation $E$ et sa section de capture $S$, est obtenue à partir de la connaissance de la loi de variation de $\tau_{\mathrm{e}}$ avec la température. La méthode la plus générale consiste à tracer un diagramme d'Arrhénius, $\operatorname{Ln}\left(\tau_{\mathrm{e}} \cdot T^{2}\right)=f(1 / T)$, qui est une droite dont la pente est proportionnelle à $E$ et l'ordonnée à l'origine proportionnelle à $S$.

Toutes les techniques de D.L.T.S. mettent en œuvre une détection de $\tau_{\mathrm{e}}$ par un filtrage plus. ou moins élaboré. L'intérêt majeur de la méthode réside dans le fait qu'elle est spectroscopique, c'est-à-dire que dans le cas le plus général où plusieurs pièges coexistent dans le matériau, un balayage en constante de temps détectée à température fixe, ou un balayage en température à constante de temps détectée fixe, permet de 
séparer les différents pièges. Comme toute technique spectroscopique, sa résolution, c'est-à-dire son pouvoir de séparer 2 niveaux de caractéristiques voisines, est un paramètre d'intérêt majeur, c'est la raison pour laquelle plusieurs équipes de chercheurs [2-4], se sont attachées à améliorer la technique de filtrage ou à en proposer de nouvelles.

Il reste que lorsqu'un piège est parfaitement résolu, s'il est possible de mesurer avec beaucoup de précision la valeur de la constante de temps détectée par le système de filtrage, pour des raisons technologiques liées entre autres à la nécessité d'un isolement électrique entre l'échantillon et la source de température, la cause majeure d'imprécision dans la détermination du couple $(E, S)$ est la mesure de $T$ pour une expérience de D.L.T.S. classique.

Effectuer une expérience de D.L.T.S., c'est, en effet, déterminer plusieurs couples de points $\left(\tau_{\mathrm{e}}, T\right)$ et la méthode la plus utilisée consiste à faire apparaître le maximum d'un "pic " de D.L.T.S., à $\tau_{\mathrm{e}}$ fixe, par un balayage en température. L'intérêt de cette méthode réside dans le fait qu'avec un seul balayage en $T$ de 77 à $300 \mathrm{~K}$, on obtient une "cartographie " à peu près complète de tous les pièges profonds $(100<E<900 \mathrm{meV}$ pour des $S$ de l'ordre de $10^{-14}$ à $10^{-16} \mathrm{~cm}^{2}$ ) du matériau étudié, alors que la méthode complémentaire ( $T$ constant, balayage en $\tau_{\mathrm{e}}$ ) ne permet généralement pas de mettre en évidence plusieurs pièges à cause de la variation exponentielle de $\tau_{\mathrm{e}}$ avec $T$.

Dans ces expériences, l'imprécision sur la mesure de $T$ est gênante car dans des conditions expérimentales même très voisines, un même niveau peut apparaître sous 2 signatures différentes, quelques dizaines de meV pour $E$ et jusqu'à 2 ordres de grandeurs pour $S$. Ceci rend la confrontation des résultats très délicate, surtout s'ils sont connus sous forme numérique. S'ils le sont sous forme de diagrammes d'Arrhénius, cette confrontation est moins critique mais elle suppose que le nombre de signatures n'excède pas une dizaine, à peu près uniformément réparties sur la partie utile du diagramme. Dans le cas typique du InP sur lequel un grand nombre de résultats expérimentaux ont été publiés ces 2 dernières années, une recherche bibliographique même sommaire permet de dénombrer au minimum une trentaine de signatures différentes et tout résultat expérimental peut alors être comparé à 3,4 ou 5 signatures déjà publiées.

Il nous paraît donc important, sinon de maîtriser les mesures de $T$, tout au moins de déterminer pour chaque piège détecté, les intervalles de confiance de la signature, c'est-à-dire du couple de valeurs $(E, S)$.

Il est évident que la mesure absolue de $T$ peut se faire actuellement avec une grande précision, mais dans tous les cas, il s'agit de la mesure au niveau de la sonde thermique. Dans un balayage en température, le gradient thermique prend alors une grande importance à cause des différences de qualité des contacts, du sens de balayage et des différences de vitesse. Même s'il est certain que toutes les dispersions de points observées sont en fait dues à des erreurs systématiques, les caúses ne pouvant être maîtrisées, ces dispersions peuvent être considérées comme à caractère aléatoire. Ceci peut être mis en évidence lors de la détermination de la signature d'un même piège à partir de diodes d'un même cristal montées sur des porte-échantillons différents.

Nous proposons, à partir d'une simulation numérique, d'approcher le problème à caractère aléatoire et d'en donner une solution empirique.

2. Description de la méthode. - Etant donné un piège de section de capture $S$ et d'énergie d'activation $E$, pour chaque constante d'émission $\tau_{\mathrm{ei}}$, on calcule sa température d'apparition $T_{\mathrm{i}}$. On définit ainsi un nombre $m$ de couples de valeurs $\left(\tau_{\mathrm{ei}}, T_{\mathrm{i}}\right)$. Expérimentalement $\tau_{\mathrm{ei}}$ est mesurée avec une très suffisante précision, par contre $T_{\mathrm{i}}$ est soumis à une fluctuation $\Delta T_{\mathrm{i}}$ autour de $T_{\mathrm{i}}$ dont la cause essentielle est provoquée par la faible qualité du contact thermique (isolement électrique) et par le fait que la température est mesurée sur le porte-échantillon et non sur la jonction elle-même et que de plus elle évolue au cours du temps lors des cycles thermiques.

Pour simuler, une expérience de D.L.T.S. nous avons admis que $\tau_{\mathrm{ei}}$ est mesurée sans erreur alors que la température mesurée est $T_{\mathrm{mi}}=T_{\mathrm{i}}+\Delta T_{\mathrm{i}}$ où $\Delta T_{\mathrm{i}}$ représente la fluctuation aléatoire de la mesure de $T_{\mathrm{i}}$. Nous avons créé un tableau de 1600 nombres pseudo-aléatoires de distribution statistique normale, centrée sur 0 et d'écart-type $1 \mathrm{~K}$, figure 1 . Pour chaque température $T_{\mathrm{i}}$ l'erreur $\Delta T_{\mathrm{i}}$ de mesure est simulée par un des nombres du tableau pris au hasard. Remarquons que pour changer l'écart-type de la distribution de 1 en $v$, il suffit de remplacer $\Delta T_{\mathrm{i}} \operatorname{par} v \Delta T_{\mathrm{i}}$.

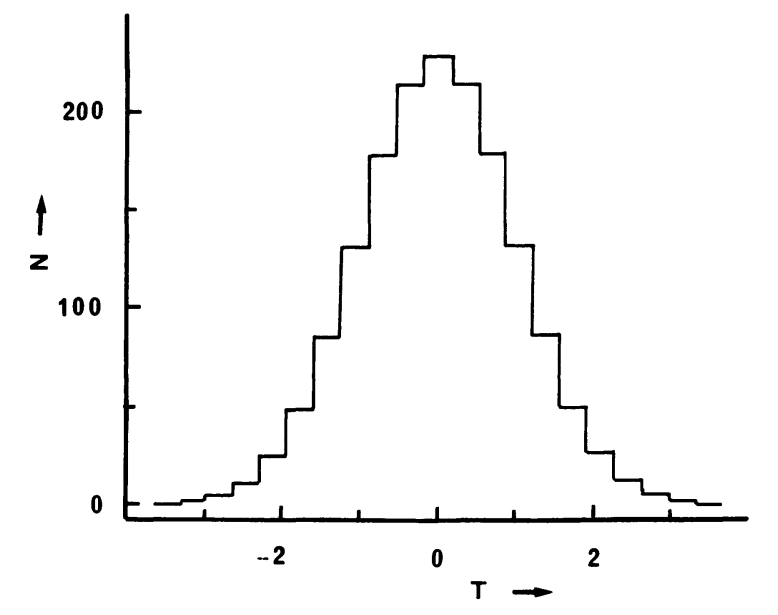

Fig. 1. - Représentation de la distribution de 1600 nombres pseudo-aléatoires en classes de 0,35.

[Graphic representation of 1600 pseudo-random number's distribution by a class width of 0.35 .] 
La figure 2 représente une telle simulation pour 5 niveaux de signatures respectives :

$$
\begin{array}{ll}
\mathrm{EL}_{1}=200 \mathrm{meV}, & S_{1}=10^{-14} \mathrm{~cm}^{2} \\
\mathrm{EL}_{2}=300 \mathrm{meV}, & S_{2}=10^{-14} \mathrm{~cm}^{2} \\
\mathrm{EL}_{3}=400 \mathrm{meV}, & S_{3}=10^{-14} \mathrm{~cm}^{2} \\
\mathrm{EL}_{4}=500 \mathrm{meV}, & S_{4}=10^{-14} \mathrm{~cm}^{2} \\
\mathrm{EL}_{5}=600 \mathrm{meV}, & S_{5}=10^{-14} \mathrm{~cm}^{2}
\end{array} .
$$

L'abaque représenté sur cette figure est constitué par les isothermes et les courbes d'égales constantes de temps. Chaque niveau est déterminé par une collection de 10 points uniformément répartis en constante de temps dans l'intervalle $10^{-3}-10 \mathrm{~s}$. Cet intervalle correspond à la réalité expérimentale d'une mesure de D.L.T.S. par transitoire de capacité. L'écart-type des $T_{\mathrm{i}}$ est de $1 \mathrm{~K}$ pour les 4 signatures, mais l'échelle hyperbolique $\left(10^{3} / T\right)$ de l'axe horizontal du diagramme cause une dispersion géométrique des points beaucoup plus grande pour les pièges excités à basse température $\left(\mathrm{EL}_{1}\right.$ ou $\left.\mathrm{EL}_{2}\right)$ que pour les pièges excités à haute température $\left(\mathrm{EL}_{4}\right.$ ou $\left.\mathrm{EL}_{5}\right)$. La détermination du couple $(E, S)$ sera par conséquent plus délicate pour un piège peu profond que pour un piège plus profond si les sections de capture restent du même ordre de grandeur.

Les couples $(E, S)$ sont obtenus ici (ou expérimentalement) à partir de la collection des $m$ points par une régression linéaire au sens des moindres carrés sur la droite d'équation $\operatorname{Ln}\left(\tau_{\mathrm{e}} \cdot T^{2}\right)=f(1 / T)$.

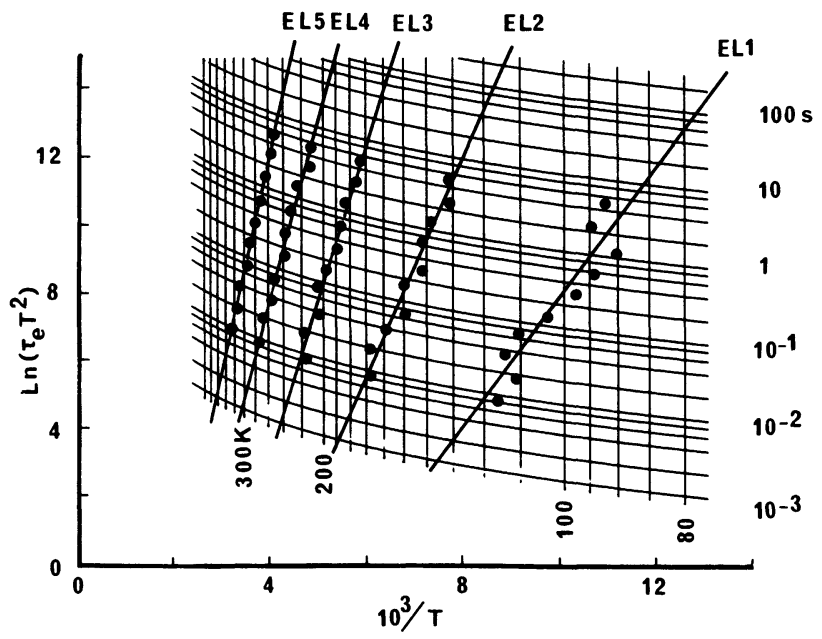

Fig. 2. - Diagrammes d'Arrhenius de 5 niveaux $\mathrm{EL}_{1}$ à $\mathrm{EL}_{5}$ d'énergies d'activation $200,300,400,500$ et $600 \mathrm{meV}$ et de sections de captures $S=10^{-14} \mathrm{~cm}^{2}$ obtenus par simulation numérique.

[Arrhenius diagrams of 5 deep levels, $\mathrm{EL}_{1}$ to $\mathrm{EL}_{5}$ having activation energies of $200,300,400,500$ and $600 \mathrm{meV}$ and capture cross-section $S=10^{-14} \mathrm{~cm}^{2}$, obtained by numerical simulation.]
Notre problème consiste donc à déterminer à partir des $m$ points les intervalles de confiance $\delta E$ de $E$ et $\delta S$ de $S$. Le nombre de ces points n'excède pratiquement jamais 30 . Pour déterminer statistiquement $\delta E$ et $\delta S$, il faut admettre que la dispersion des $T_{\mathrm{mi}}$ obéit à une loi de Student, la statistique gaussienne n'étant raisonnablement applicable que lorsque la collection des points atteint une centaine [5].

Si on pose $X_{\mathrm{i}}=10^{3} / T_{\mathrm{mi}}$ et $Y_{\mathrm{i}}=\operatorname{Ln}\left(\tau_{\mathrm{ei}} \cdot T_{\mathrm{mi}}^{2}\right)$, on définit (5) pour $m$ points :

- Le coefficient de corrélation de la régression $r=$

$\frac{\sum_{m} X_{\mathrm{i}} \cdot Y_{\mathrm{i}}-(1 / m) \sum_{m} X_{\mathrm{i}} \cdot \sum_{m} Y_{\mathrm{i}}}{\left\{\left(\sum_{m} X_{\mathrm{i}}\right)^{2}-\sum_{m} X_{\mathrm{i}}^{2} / m\right\}^{1 / 2}\left\{\left(\sum_{m} Y_{\mathrm{i}}\right)^{2}-\sum_{m} Y_{\mathrm{i}}^{2} / m\right\}^{1 / 2}}$

- Les moyennes arithmétiques $\bar{X}$ et $\bar{Y}$ des distributions des $X_{\mathrm{i}}$ et des $Y_{\mathrm{i}}$.

- La variance des $X_{\mathrm{i}}$

$$
S_{x}^{2}=1 / m \sum_{m}\left(X_{\mathrm{i}}-\bar{X}\right)^{2} .
$$

- La variance des $Y_{\mathrm{i}}$ liés aux $X_{\mathrm{i}}$

$$
S_{y x}^{2}=1 / m\left(1-r^{2}\right) \sum_{m}\left(Y_{i}-\bar{Y}\right)^{2} .
$$

Alors on obtient les probabilités : $\delta E:$

$-p_{1}$ pour que l'intervalle de confiance de $E$ soit

$$
p_{1}=\frac{\delta E}{2\left(1-r^{2}\right)^{1 / 2}}(m-2)^{1 / 2},
$$

$-p_{2}$ pour que l'intervalle de confiance de Log $S$ soit $\delta(\log S)$

$$
p_{2}=\frac{\delta(\log S)}{S_{y x}\left(1+\frac{1}{m}-\frac{\bar{X}^{2}}{m S_{x}^{2}}\right)^{1 / 2}}
$$

$p_{1}$ et $p_{2}$ sont des probabilités déterminées par une distribution de Student à $m-2$ degrés de liberté. En admettant que l'intervalle de confiance est déterminée par une probabilité $p_{0}=1-10^{-4}$, les équations (5) et (6) permettent de calculer à partir d'une table de Student $\delta E$ et $\delta(\log S)$.

Remarquons que $\delta(\log S)$ représente le nombre d'ordres de grandeur sur la précision de $S$.

3. Résultats de la simulation. - Nous avọns calculé, pour chacun des niveaux $\mathrm{EL}_{1}$ à $\mathrm{EL}_{5}$ les diagrammes d'Arrhénius correspondant à des collections de 25, 15 et 10 points avec des distributions en $T_{\mathrm{i}}$ de $0,5,1$, $1,5,2$ et 2,5 K. Nous en avons donné un exemple sur la figure 1 . Nous avons consigné sur les tableaux I et II des exemples de déterminations de $\delta E$ et $\delta(\log S)$ 
Tableau I. - Résultats numériques des simulations relatives à un centre de signature $E=600 \mathrm{meV}$, $S=10^{-14} \mathrm{~cm}^{2}$. $m$ est le nombre de points, $r$ le coefficient de corrélation de chaque régression, $\delta E$ l'intervalle de confiance de la détermination de $E, \delta \log (S)$ l'intervalle de confiance en nombre d'ordres de grandeur de la détermination de $S$.

[Numerical results after simulations relative to a centre, $E=600 \mathrm{meV}$, and $S=10^{-14} \mathrm{~cm}^{2} . m$ is the number of experimental points, $r$ the coefficient of correlation for each fitting, $\delta E$ the confidence interval in the determination of $E$ and $\delta(\log S)$ is the confidence interval, as order of magnitude, in the determination of $S$.]

\begin{tabular}{ccccc}
\hline$m$ & $v \cdot \Delta T_{\mathrm{i}}(\mathrm{K})$ & $r(\%)$ & $\delta E(\mathrm{meV})$ & $\delta \log (S)$ \\
\hline 25 & - & - & - & - \\
25 & 0,5 & 99,9 & 4 & 0,4 \\
25 & 1 & 99,8 & 5 & 0,5 \\
25 & 1,5 & 99,2 & 12 & 1 \\
25 & 2 & 99,3 & 12 & 1 \\
15 & 2,5 & 98,6 & 16 & 1,4 \\
15 & 0,5 & 99,9 & 3 & 0,3 \\
15 & 1 & 99,7 & 11 & 0,7 \\
15 & 1,5 & 99,6 & 13 & 0,9 \\
15 & 2 & 99 & 20 & 1,3 \\
10 & 2,5 & 98,5 & 24 & 1,7 \\
10 & 0,5 & 99,8 & 7 & 0,4 \\
10 & 1 & 99,8 & 13 & 0,7 \\
10 & 1,5 & 99,2 & 27 & 1,5 \\
10 & 2 & 99,4 & 24 & 1,3 \\
& 2,5 & 96 & 51 & 3,4 \\
\hline
\end{tabular}

Tableau II. - Résultats numériques des simulations relatives $\grave{a}$ un centre de signature $E=200 \mathrm{meV}$, $S=10^{-14} \mathrm{~cm}^{2}$. Les paramètres du tableau sont définis dans la légende du tableau I.

[Numerical results of simulations relative to a centre, $E=200 \mathrm{meV}$ and $S=10^{-14} \mathrm{~cm}^{2}$. The definitions of parameters are the same as those of table I.]

\begin{tabular}{ccccc}
\hline$m$ & $v . \Delta T_{\mathrm{i}}(\mathrm{K})$ & $r(\%)$ & $\delta E(\mathrm{meV})$ & $\delta \log (S)$ \\
$\overline{2}$ & - & - & - & - \\
25 & 0,5 & 99 & 7 & 2 \\
25 & 1 & 98 & 12 & 4,2 \\
25 & 1,5 & 97 & 24 & 5,6 \\
15 & 2 & 93 & 35 & 8,7 \\
15 & 1 & 99,6 & 12 & 2,3 \\
15 & 1,5 & 98,9 & 22 & 4 \\
15 & 2 & 94 & 50 & 9,6 \\
10 & 0,5 & 99,4 & 25 & 3,4 \\
10 & 1 & 98,7 & 34 & 5,2 \\
10 & 1,5 & 96 & 57 & 9 \\
10 & 2 & 92 & 86 & 13 \\
\hline
\end{tabular}

relatifs à $E_{1}$ et $\mathrm{EL}_{5}$. Pour $\mathrm{EL}_{1}$ compte tenu des valeurs excessives de $\delta(\log S)$ nous n'avons pas simulé de collection de points pour un écart-type de $2,5 \mathrm{~K}$.

4. Discussion. - Comme nous l'avions prévu lors du simple examen de la figure 1 , les incertitudes $\delta T_{\text {i }}$ peuvent être catastrophiques pour les pièges peu profonds. C'est ce qui ressort du tableau II où une distribution d'écart-type $2 \mathrm{~K}$ conduit malgré un coefficient de corrélation de $92 \%$ à des résultats qu'il conviendrait d'écrire :

$$
157 \mathrm{meV}<E<243 \mathrm{meV}
$$

mais surtout

$$
3 \times 10^{-21} \mathrm{~cm}^{2}<S<3 \times 10^{-8} \mathrm{~cm}^{2} .
$$

Ce dernier résultat est sans signification physique.

Nous avons appliqué, cette procédure expérimentale sur un piège de l'InP rapporte par H. Lim [6] sous la dénomination « $E_{5}$ " figure 3. Pour 25 points expérimentaux et un coefficient de corrélation de $94 \%$, on obtient :

$$
\begin{gathered}
395 \mathrm{meV}<E<432 \mathrm{meV}, \\
2 \times 10^{-18} \mathrm{~cm}^{2}<S<7 \times 10^{-15} \mathrm{~cm}^{2} .
\end{gathered}
$$

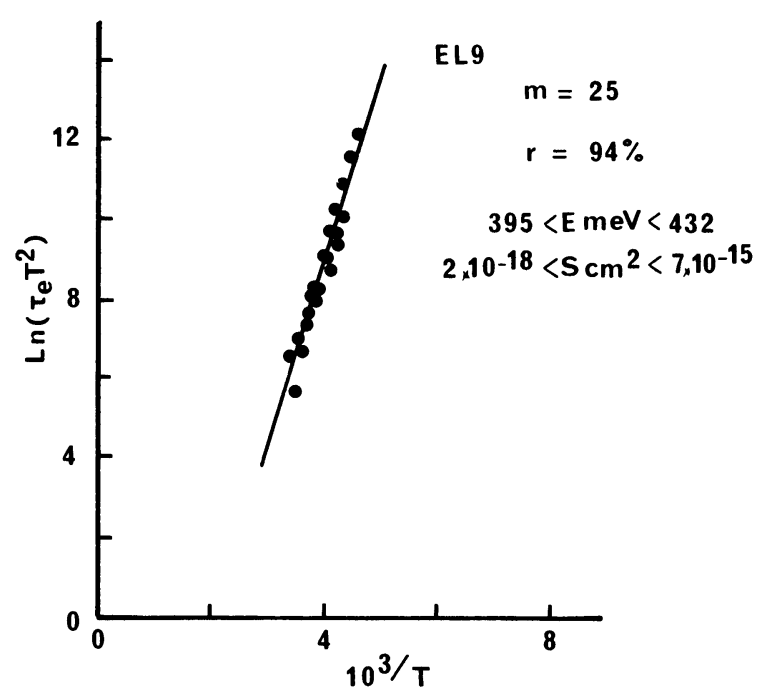

Fig. 3. - Diagramme d'Arrhenius de « $\mathrm{E}_{5} »$ de H. Lim [6].

[Arrhenius diagram of « $E_{5}$ " level taken from the results of reference [6].]

5. Approche empirique. - Sur la figure 4, nous avons tracé les intervalles de confiance $\delta E$ de $E$ en fonction du coefficient de corrélation.

Remarquons que :

- $\delta E$ est très peu dépendant de $E$,

- $\delta E$ dépend essentiellement de $r$ et de $m$.

Comme il ne s'agit que de déterminer une estimation de précision de mesure, on peut négliger la variation en $E$ et écrire $E=f(r, m)$. 


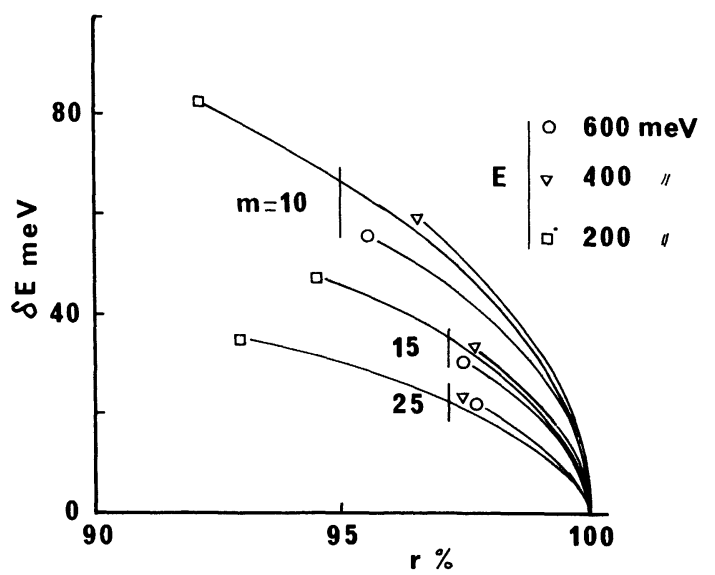

Fig. 4. - Intervalles de confiance $\delta E$ de détermination de $E$ en fonction du coefficient de correlation $r$ de chaque régression linéaire pour 3 niveaux, le nombre de points $m$ est pris comme paramètre.

[Confidence intervals $\delta E$ in the determination of $E$ as a function of the coefficient of correlation and of each least square fitting for 3 deep levels, the number of points $m$ is taken as a parameter.]

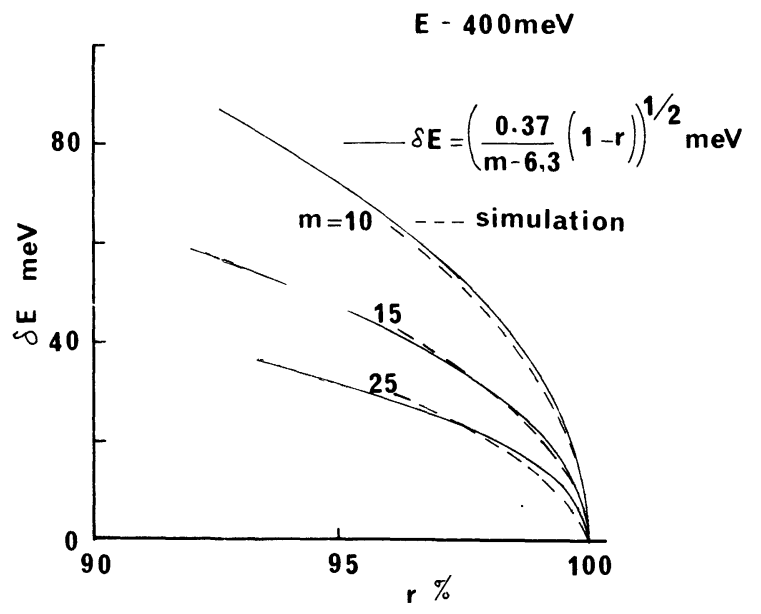

Fig. 5. - Comparaison de la détermination de $\delta E$ par la relation empirique (7) avec les résultats de la simulation.

[Comparison between the determination of $\delta E$ by empirical formula (7) and the results of simulation.]

Les courbes théoriques de la figure 5 montrent que l'approximation de $\delta E$ par la relation :

$$
(\delta E)_{\mathrm{meV}}=10^{3} \frac{0,37}{m-6,3}(1-r)^{1 / 2}
$$

est très convenable mais en toute rigueur elle n'est

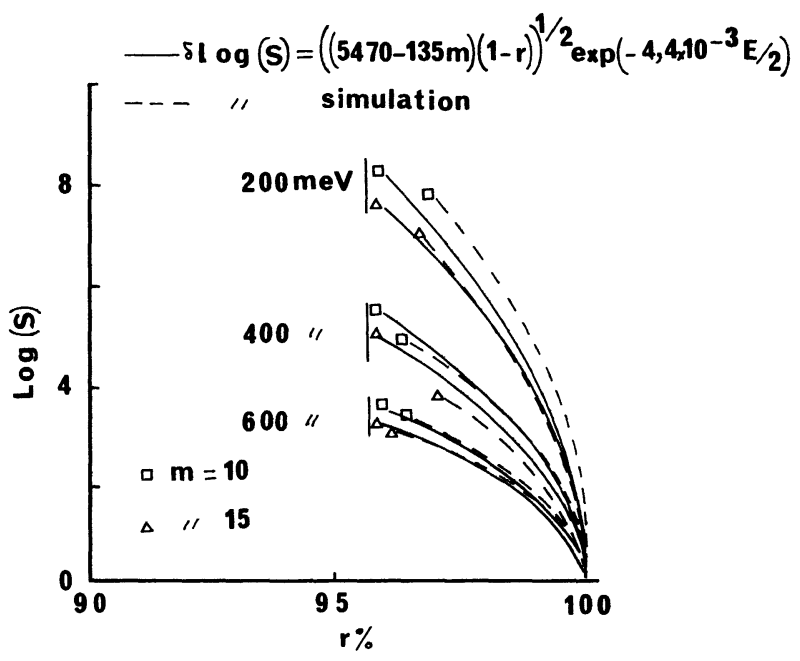

Fig. 6. - Comparaison de la détermination de $\delta(\log S)$, intervalle de confiance en nombre d'ordres de grandeur de $S$, par la relation empirique (8) avec les résultats de la simulation.

[Comparison between the determination of $\delta(\log S)$ and the results of simulation. The confidence intervals are taken by orders of magnitude of $S$.]

valable que pour :

$$
\begin{aligned}
0,95 & <r<1 \\
200 & <E<600 \mathrm{meV} \\
10 & <m<25 .
\end{aligned}
$$

Par la même procédure et pour des sections de captures de l'ordre de $10^{-14} \mathrm{~cm}^{2}$, avec les mêmes limitations en $r, E$ et $m$ on peut approximer $\delta(\log S)$ par :

$$
\begin{aligned}
& \delta(\log S)=(5470-135 m)^{1 / 2}(1-r)^{1 / 2} \\
& \exp -\left(4,4 \times 10^{-3} E / 2\right)
\end{aligned}
$$

où $E$ est exprimé en meV.

La figure 6 illustre cette approche empirique de $\delta(\log S)$.

6. Conclusion. - Nous avons montré par quelle procédure on peut déterminer théoriquement les intervalles de confiance $\delta E$ de l'énergie d'activation et $\delta(\log S)$ nombre d'ordres de grandeurs de la section de capture dans une expérience de D.L.T.S.

Nous en avons donné un exemple expérimental sur InP et montré que pour des énergies d'activation comprises entre 200 et $600 \mathrm{meV}$, un nombre de points de 10 à 25 et un coefficient de corrélation raisonnable $(r>93 \%)$ on peut obtenir une valeur très satisfaisante des intervalles de confiance en les calculant à partir des relations empiriques (7) et (8). 


\section{Bibliographie}

[1] Lang, D. V., J. Appl. Phys. 45 (1974) 3023.

[2] Day, D. S., Tsai, M. Y., Streetman, B. G. and Lang, D. V., J. Appl. Phys. 50 (1979) 5093.

[3] Miller, G., Ramirez, J. V. and Robinson, D. A. H., J. Appl. Phys. 46 (1975) 2638.

[4] Crowell, C. R. and Alipanahi, S., Solid State Electron. 24 (1980) 25.

[5] SpIEgew, R., Théorie et application de la statistique, série Shaum édité par (McGraw-Hill).

[6] Lim, H., Accepté pour publication dans J. Appl. Phys. (1981). 